\title{
Concealment of Chaos Time-Delay Signature in Three-Cascaded Vertical- Cavity Surface-Emitting Lasers
}

Hong, Yanhua; Quirce, Ana; Wang, Bingjie; Ji, Songkun; Panajotov, Krassimir; Spencer, Paul

\section{IEEE Journal of Quantum Electronics}

DOI:

10.1109/JQE.2016.2587099

Published: 01/08/2016

Peer reviewed version

Cyswllt i'r cyhoeddiad / Link to publication

Dyfyniad o'r fersiwn a gyhoeddwyd / Citation for published version (APA):

Hong, Y., Quirce, A., Wang, B., Ji, S., Panajotov, K., \& Spencer, P. (2016). Concealment of Chaos Time-Delay Signature in Three-Cascaded Vertical-Cavity Surface-Emitting Lasers. IEEE Journal of Quantum Electronics, 52(8). https://doi.org/10.1109/JQE.2016.2587099

\footnotetext{
Hawliau Cyffredinol / General rights

Copyright and moral rights for the publications made accessible in the public portal are retained by the authors and/or other copyright owners and it is a condition of accessing publications that users recognise and abide by the legal requirements associated with these rights.

- Users may download and print one copy of any publication from the public portal for the purpose of private study or research.

- You may not further distribute the material or use it for any profit-making activity or commercial gain

- You may freely distribute the URL identifying the publication in the public portal?
}

Take down policy

If you believe that this document breaches copyright please contact us providing details, and we will remove access to the work immediately and investigate your claim. 


\title{
Concealment of Chaos Time-Delay Signature in Three-Cascaded Vertical-Cavity Surface-Emitting Lasers
}

\author{
Yanhua Hong, Member, IEEE, Ana Quirce, Bingjie Wang, Songkun. Ji, Krassimir Panajotov, \\ Member, IEEE, Paul S. Spencer
}

\begin{abstract}
The time-delay signature and chaos bandwidth in three-cascaded vertical-cavity surface-emitting lasers (VCSELs) has been investigated experimentally. A peak value of autocorrelation coefficient at the feedback round trip time and the ratio between this peak value and its background are used to quantitatively identify the time-delay (TD) signature of chaos. A new concept - peak to side-peak ratio is introduced for better quantification of the TD signature concealment. The peak to side-peak ratio is defined as the ratio between the peak value of autocorrelation coefficient at the TD and the peak value at a delay time other than the TD and zero delay time. Three injection cases, small bandwidth, intermediate bandwidth and wide bandwidth of the injecting chaos signals, have been used to study the effect of the bandwidth of the injecting chaos on the TD concealment. The experimental results show that the time-delay signature can be totally concealed in the slave laser subject to the intermediate bandwidth of chaotic optical injection over a wide frequency detuning range.
\end{abstract}

Index Terms-Chaos, optical feedback, time delay (TD), vertical-cavity surface-emitting lasers (VCSEL).

\section{INTRODUCTION}

$\mathrm{O}$ PTICAL chaos has many potential applications, such as chaos-based optical communications [1-7], physical random number generators [8-10], optical time-domain reflectometry [11-13], chaotic lidar [14], etc. Optical chaos can be generated in many laser systems, such as solid-state lasers [15], gas lasers [16], fiber lasers [17] and semiconductor lasers [1-10]. Semiconductor lasers are the most commonly used for generated optical chaos since they are readily available and

Manuscript received May 6, 2016. This work was supported in part by the Sêr Cymru National Research Network in Advanced Engineering and Materials.

Y. Hong, S. K. Ji and P. S. Spencer are with School of Electronic Engineering, Bangor University, Gwynedd LL57 1UT, Wales, UK. (e-mail: y.hong@bangor.ac.uk; eep40a@bangor.ac.uk; p.spencer@bangor.ac.uk)

A. Quirce and K. Panajotov are with Department of Applied Physics and Photonics, Brussels Photonics Team, Vrije Universiteit Brussel, Pleinlaan 2, 1050, Brussels, Belgium. (e-mail: aquirce@b-phot.org; kpanajot@b-phot.org)

K. Panajotov is also with Institute of Solid State Physics, 72 Tzarigradsko, Chausse Blvd., 1784 Sofia, Bulgaria.

B. Wang is with Key Laboratory of Advanced Transducers and Intelligent Control System, Ministry of Education and Shanxi Province, and College of Physics and Optoelectronics, Taiyuan University of Technology, Taiyuan 030024, China. (e-mail: wangbingjie@ tyut.edu.cn). easy to operate. According to a recent review about physics and applications of laser diode chaos, there are two types of chaotic system in semiconductor lasers [18]. In one type of chaotic system, chaos can be generated without applying additional force or parameter modulation. A typical example is the chaos from a solitary quantum-dot vertical-cavity surface-emitting laser (VCSEL) [19]. The physical mechanism behind this polarization chaos in the free running VCSEL is the nonlinear coupling between two elliptically polarized modes. The chaos generated under this mechanism has low bandwidth and low dimension. However, such a chaos with low bandwidth and low dimension can also be employed to generate high bit rate $(>100 \mathrm{~Gb} / \mathrm{s})$ random bits due to its distinct polarization dynamics properties which result in the growth of dynamical entropy [20]. Another type of chaotic system is that chaos generated in semiconductor laser by adding disturbance, such as, feedback [1-5], modulation [21] or optical injection [14]. Optical feedback is the most common method to obtain chaos. For the applications of chaos generated by optical feedback, there are two important parameters - chaos bandwidth and time delay (TD) signature. A broad bandwidth without TD signature is preferred for most application. Narrow chaos bandwidths will limit message transmission rates in chaos-based communication, the bit rate of random number generation and the range resolution in optical time-domain reflectometry and chaotic lidar [10,14]. The TD signature of chaos is a recurrence feature induced by the optical round trip in the external cavity. The TD signature may provide the opportunity for an eavesdropper to extract a key parameter, which may compromise the security of chaotic optical communications. The TD signature reduces the randomness of the chaotic optical signal and affects the symmetrical distribution of random bits. The TD signature may also cause a range of unambiguous detections in optical time-domain reflectometry and chaotic lidar.

Several studies have considered either the bandwidth enhancement [22-26] or the TD signature suppression [27-32]. There are few reports which try to optimize both the bandwidth enhancement and TD signature suppression [33-35], however, the TD signature has not been totally concealed. Time delay concealment and bandwidth enhancement of chaos have only been achieved using three DFB lasers [36]. A recent numerical simulation result predicted that wideband chaos with TD 
concealment, over a wider parameters regime, can be achieved using three-cascaded VCSELs [37]. However, to the best of our knowledge, no experimental work has been undertaken to study both the time delay signature and bandwidth of chaos in three-cascaded VCSELs. Moreover, previous works [36-37] did not investigate the effect of the bandwidth of the injecting chaos on the TD signature.

In this work, we have experimentally examined the TD signature and the bandwidth of chaos in three-cascade VCSELs system. Due to the limitations of the detection of the polarization-resolved outputs in our all-fiber setup, the experimental study has been focused on the effect of the injecting beam bandwidth on the TD signature and the chaos bandwidth of the total power of the slave laser. Three cases of the chaotic optical injection - small bandwidth, intermediate bandwidth and high bandwidth of the injecting signal, have been studied. The small, intermediate and higher bandwidths are related to the minimum conventional bandwidth of the injecting chaotic signal. The conventional bandwidth is defined as the frequency difference between DC and the frequency which contains $80 \%$ of the power [23-25]. In order to classify the different behaviours observed experimentally, small, intermediate and high bandwidth of injecting chaos are defined as: when the conventional bandwidth of the injecting chaos is less than 1.2 times the minimum conventional bandwidth, we refer it as the small bandwidth; when the conventional bandwidth of the injecting chaos is more than 2.1 times the minimum conventional bandwidth, we consider it as the high bandwidth; in between, it is noted as the intermediate bandwidth.

The remainder of this paper is organized as follows. The experimental setup and the operation parameters are described in Section II, followed by the TD signature analysis methods in Section III. The experimental results and discussion for two VCSELs system and three-cascaded VCSELs system are in section IV. Finally, in section V, conclusions are drawn based on the results obtained.

\section{EXPERIMENTAL SETUP}

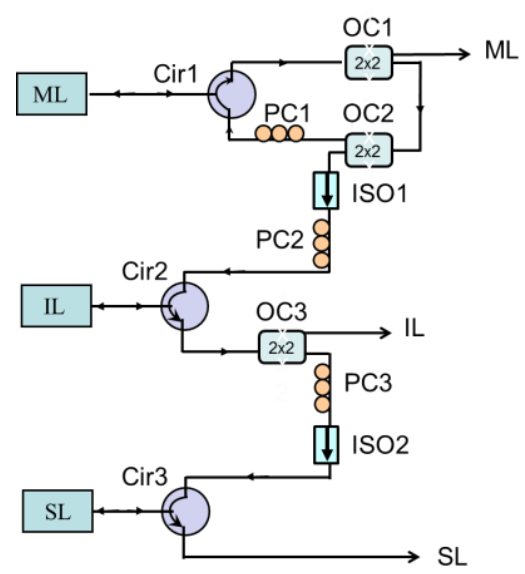

Fig. 1. The experimental setup. ML- master laser; IL- intermediate laser; SL - slave laser; Cir-optical circulator; OC-optical coupler; ISO-optical isolator; PC-polarization controller.
The all-fiber experimental setup is shown in Fig. 1. Three commercial VCSELs with lasing wavelength of approximately $1550 \mathrm{~nm}$ (RayCan fibre pigtailed VCSEL) were used as master laser (ML), intermediate laser (IL) and slave laser (SL). These VCSELs were driven by ultra-low noise current sources and were temperature controlled to an accuracy of $0.01^{\circ} \mathrm{C}$. The threshold currents of the ML, IL and SL were $1.7 \mathrm{~mA}, 1.75 \mathrm{~mA}$ and $1.7 \mathrm{~mA}$, respectively and a polarization switch occurred at bias currents of $7.1 \mathrm{~mA}, 5.75 \mathrm{~mA}$ and $4.49 \mathrm{~mA}$ for the ML, IL and SL, respectively. Optical isolator 1 and 2 (ISO1 and ISO2) were used to ensure unidirectional coupling from the ML to the IL and from the IL to the SL, respectively. The ML was subject to optical feedback and was rendered chaotic. Polarization controller (PC1) in the feedback loop was used to control the feedback polarization to achieve maximum chaotic bandwidth in the ML. A fraction of the chaotic ML output was injected into the IL via ISO1 and a polarization controller (PC2). PC2 was used to control the polarization of the optical injection beam to obtain complex chaos in the IL. The SL was subject to optical injection from the chaotic IL and entered chaotic dynamics.

In this paper, the feedback ratio is defined as the ratio of the optical feedback power and the ML's free-running output power. The optical feedback power is measured just before it is fed back into the ML. The injection power is measured just before the beams are injected into the IL or SL and the injection ratio is defined as the ratio of the injection power and the output power of the stand-alone IL or SL. The frequency detuning between the ML and IL $\left(\Delta \mathrm{f}_{1}\right)$ is defined as the frequency difference between the free-running frequency of the ML and the free-running frequency of the IL. The frequency detuning between the IL and $\mathrm{SL}\left(\Delta \mathrm{f}_{2}\right)$ is defined as the frequency difference between the free-running frequency of the IL and the free-running frequency of the SL. The detunings of $\Delta \mathrm{f}_{1}$ and $\Delta \mathrm{f}_{2}$ are achieved by changing the temperature of the ML and the SL, respectively. The output of the VCSEL was detected by 12 $\mathrm{GHz}$ bandwidth photo-detectors (New Focus 1554-B) and recorded using a $30 \mathrm{GHz}$ bandwidth $\mathrm{RF}$ spectrum analyzer (Anritsu MS2667C) and 4GHz bandwidth oscilloscope (Tektronix TDS7404). The sampling rate of the oscilloscope is set at $10 \mathrm{GS} / \mathrm{s}$ and 200000 samples are recorded for each time trace, therefore the duration of each time trace is $20 \mu \mathrm{s}$.

In order to operate the VCSELs well below polarization switching current, also to match the wavelengths of these three VCSELs, the bias currents of the ML, IL and SL were fixed at $3.5 \mathrm{~mA}, 3.3 \mathrm{~mA}$ and $2.34 \mathrm{~mA}$, respectively. At these bias currents, the VCSELs operated in single mode regimes. The maximum feedback ratio of the ML was used in the experiment with a value of $-9.5 \mathrm{~dB}$. The maximum injection ratios of the IL and SL were also applied with the values of $-9.0 \mathrm{~dB}$ and -4.0 $\mathrm{dB}$, respectively. The effect of the feedback ration and injection power on the TD signature and the bandwidth of chaos were not investigated because of the lower output power of VCSELs. The feedback round trip time of the ML was about $76.2 \mathrm{~ns}$. The flight time from the ML to the IL and the IL to the SL were about $81.3 \mathrm{~ns}$ and $76.2 \mathrm{~ns}$, respectively. 


\section{TIME DELAY SIGNATURE ANALYSIS METHODS}

The common methods to quantify the TD signature are autocorrelation function (ACF), delayed mutual information and permutation entropy [27-39]. For the time being, most of the studies on the TD signature analyze the intensity time series, whereas a few reports investigate the phase time series $[37,40]$. The investigation of phase time series will be very interesting though it is out of scope of this paper. And we used the $(\mathrm{ACF})$ to identify the TD signature. The autocorrelation coefficient is labelled as $\mathrm{C}$ and is defined as follows

$$
C(\Delta t)=\frac{<[I(t+\Delta t)-<I(t+\Delta t)>][I(t)-<I(t)>]>}{\sqrt{<[I(t+\Delta t)-<I(t+\Delta t)>]^{2}><[I(t)-<I(t)>]^{2}>}}
$$

where $\Delta t$ is the delay time, $I(t)$ is the output intensity of the VCSEL, <-> denotes time average. The peak value $\left(C_{P}\right)$ of autocorrelation coefficient at the feedback round trip time is commonly used to quantify the TD signature. The peak value at the feedback round trip time can be expressed as:

$$
C_{p}=\max |C(\Delta t)| \Delta t \in v_{1}\left(\tau_{d}\right)
$$

where $\tau_{\mathrm{d}}$ is the feedback round trip time. The measured TD peak value may not be located exactly at $\tau_{\mathrm{d}}$. If the measured peak value is located in the interval $v_{l}\left(\tau_{d}\right)=\left(\tau_{d}-\tau_{d} \times r_{l}, \tau_{d}+\tau_{d}\right.$ $\left.\times r_{l}\right)$; this peak value is considered as the peak value at TD. According to the experimental data, $2 \%$ is selected as the value of $r_{1}$.

Some reports use the peak signal to mean ratio $\left(R_{c}\right)$ to quantify the TD signature [38]. In this paper, $R_{c}$ is slightly extended for a better indication of the peak value to the background ratio. Here $R_{c}$ is defined as the ratio between $C_{p}$ and the averaged absolute autocorrelation coefficient over the interval $v_{2}=\left(\tau_{d}-\tau_{d} \times r_{2}, \tau_{d}-\tau_{d} \times r_{l}\right) \cup\left(\tau_{d}+\tau_{d} \times r_{1}, \tau_{d}+\tau_{d} \times r_{2}\right)$ around the feedback round trip time delay time, but the interval $v_{l}\left(\tau_{d}\right)$ is not included. $5 \%$ is selected as the value of $r_{2}$ :

$$
R_{C}=\frac{\max |C(\Delta t)| \Delta t \in v_{1}\left(\tau_{d}\right)}{\left\langle|C(\Delta t)| \Delta t \in v_{2}\right\rangle}
$$

If there is more than one peak in the ACF curve and the peak at the other delay time is higher than the peak at the time delay, the TD signature would be difficult to identify, which provides better security for chaotic optical communications. Here we introduce a new parameter, the peak to side-peak ratio, to identify the TD signature. The peak to side-peak ratio $\left(R_{p p}\right)$ is defined as the ratio of $\mathrm{C}_{\mathrm{P}}$ and the peak value at a delay time $\Delta \mathrm{t}$ other than the feedback round trip time and the zero delay time:

$$
R_{P P}=\frac{\max |C(\Delta t)| \Delta t \in v_{1}\left(\tau_{d}\right)}{\max |C(\Delta t)| \Delta t \notin 0, v_{1}\left(\tau_{d}\right)}
$$

If $R_{P P} \leq 1$, the peak at TD is equal to or lower than the peak at the other delay time. In this case, the TD signature has been disguised.

\section{EXPERIMENTAL RESULTS AND DISCUSSION}

Both autocorrelation coefficient and permutation entropy (PE) [41] have been calculated to quantify the TD signature. The definition and parameters selected for PE can be found in [31], where the embedding dimension $M$ is set as 4._Fig. 2 shows the time traces, autocorrelation coefficient curves, permutation entropy curves and the radio-frequency (RF) power spectra of chaos generated in the ML, IL and SL. The top, middle and bottom rows are for the ML, IL and SL, respectively. The first, second, third and fourth columns are for the time traces, the autocorrelation coefficient curves, permutation entropy curves and the RF power spectra, respectively. The frequency detunings are $\Delta \mathrm{f}_{1}=-3.7 \mathrm{GHz}$ and $\Delta \mathrm{f}_{2}=8.3 \mathrm{GHz}$. Fig. 2(b1) shows a clear TD signature at 76.2ns. After optical injection, the TD signature in the IL has been significantly reduced, as shown in Fig. 2(b2), however, the TD signature can still be identified at the delay time of $76.2 \mathrm{~ns}$. When the chaos with the suppressed TD signature is injected into the SL, the TD signature is further reduced. In Fig. 2(b3), the TD signature is totally blended into the noise, the TD signature has been completed concealed. Fig. 2(c1) also shows a clear TD signature at $76.2 \mathrm{~ns}$ and its integer fractions, however, the TD signature is been difficult to identify in Fig. 2(c2), indicating that the permutation entropy calculated by the parameters we selected is less sensitive than the ACF in identifying the TD. In the following, only the data extracted from the ACF are used to quantify the TD signature. The RF power spectra of the chaotic signal with the same injection parameters are also examined and shown in the fourth column of Fig. 2. For all the RF power spectra shown in this paper, the noise has been removed. Fig. 2(d1) is a typical chaotic spectrum induced by optical feedback. The peak at about 1.6 $\mathrm{GHz}$ in the spectrum is related to the laser relaxation oscillation frequency. From the power spectrum of the IL in Fig. 2(d2), it is clearly discernible that the power in the higher frequencies components increases compared with that of the ML, i.e. the bandwidth of chaos is increased. For Fig. 2(d3), the chaotic bandwidth is further increased. These results clearly demonstrate that three-cascaded VCSELs system can achieve broad bandwidth chaos with total concealment of the TD signature.
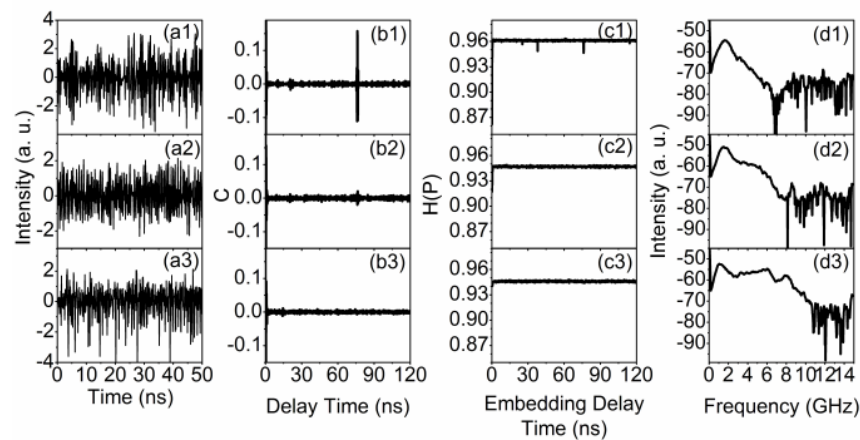

Fig. 2 The time traces (first column), autocorrelation coefficient curves (second column), permutation entropy curves (third column) and RF power spectra (fourth column) of chaotic signal. The top, middle and bottom rows are for the ML, IL and SL, respectively. 
Although the emphasis of this manuscript is TD signature, chaos bandwidth is also calculated in the following results because it is an important parameter for chaos applications. Two methods have been used to evaluate chaos bandwidth. One is the conventional method, which has been defined above. Another method is the effective bandwidth, which sums up only those discrete spectral segments of the chaos power spectrum accounting for $80 \%$ of the total power [42].

\section{A. Two VCSELs System}

For comparison purposes, the TD signature and the bandwidth of chaos in a two VCSELs system have been measured. Fig. 3 shows the chaos bandwidth and the TD signature of the IL as a function of the frequency detuning $\Delta \mathrm{f}_{1}$. The conventional and effective bandwidths of the chaotic signal in the ML are about $2.4 \mathrm{GHz}$ and $2.3 \mathrm{GHz}$, respectively. Fig. 3(a) shows that both the conventional bandwidth and effective bandwidth of the chaotic signal in the IL increases significantly outside zero frequency detuning, which is similar to that in [34]. The minimum conventional and effective bandwidths are $2.4 \mathrm{GHz}$ and $2.0 \mathrm{GHz}$, respectively. The effective bandwidths are slightly smaller than the conventional bandwidths. Also the effective bandwidth starts to drop for larger negative frequency detuning. Fig. 3(b) plots the peak value $C_{p}$ of the autocorrelation coefficient at the feedback round trip time. For comparison, the $\mathrm{C}_{\mathrm{p}}$ values of the $\mathrm{ML}$ are also calculated and displayed in Fig. 3(b). The result shows that $\mathrm{C}_{\mathrm{p}}$ value of the IL is greatly suppressed in comparison with those of the ML. This TD signature suppression can be attributed to the chaos-pass filter effect [34]. A small difference between these results and those in [34] is that the suppression of the TD signature near zero frequency detuning is less. This may be caused by higher injection ratio, where the IL is locked by the ML. The peak value to the background ratio $R_{c}$ has been calculated and shown in Fig.3(c), the trend is similar to Fig. $3(b)$, the suppression of TD signature is less near zero frequency detuning and large positive frequency detuning. Fig. 3(d) displays the peak to side-peak ratio $R_{p p}$ of chaotic signal from the IL. The results show that the values of $R_{p p}$ are higher than 1 for most of the frequency detuning, so the TD signature is not concealed.
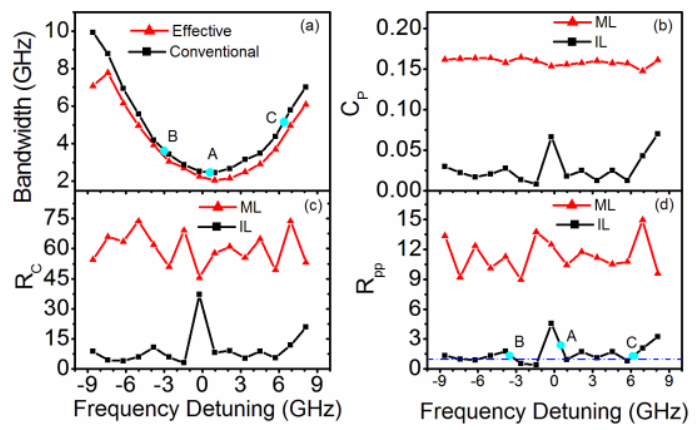

Fig. 3 Two VCSELs system. The bandwidth and the TD signature of the chaotic signal of the IL as a function of the frequency detuning $\Delta f_{1}$ between ML and IL. (a) conventional and effective chaos bandwidths; (b) $\mathrm{C}_{\mathrm{P}}$; (c) $\mathrm{R}_{\mathrm{c}}$; (d) $\mathrm{R}_{\mathrm{PP}}$.

\section{B. Three-Cascaded VCSELs System}

In a three-cascaded VCSELs system, the SL was operated in chaotic dynamics regime due to optical injection from the chaotic IL. The effect of the bandwidth and TD signature of the injecting chaos on the TD suppression and the bandwidth enhancement of the SL has been investigated. There are three cases: 1) the injecting chaos has a narrow bandwidth and slightly higher TD signature chaos; 2) the injecting chaos has intermediate bandwidth and low TD signature and 3) the injecting chaos has broad bandwidth and low TD signature.

1) Case I - narrow bandwidth and slightly higher TD signature of the injecting chaos
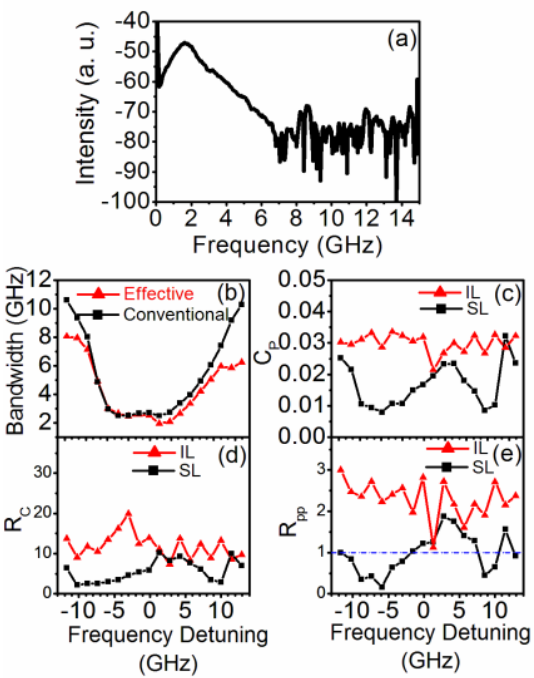

Fig. 4 Three VCSELs system with narrow bandwidth and slightly higher TD signature of the injecting chaos. (a) The RF power spectrum of the IL; (b) conventional and effective chaos bandwidths of the SL and (c-e) the TD signature of the chaotic signal as a function of the frequency detuning $\Delta \mathrm{f}_{2}$ between IL and SL. (c) $C_{P}$; (d) $R_{c}$; (e) $R_{P P}$.

In this case, the conventional chaos bandwidth from the IL is about $2.4 \mathrm{GHz}$, which is very close to the minimum bandwidth, as shown at point A in Fig. 3(a). Fig. 4(a) shows the power spectrum of the injecting chaotic signal. This power spectrum of the injecting chaotic signal is almost the same to that of the ML. But the TD signature of this chaos is much lower than that of the ML, as shown in Fig. 3(b). Fig. 4(b) shows both the conventional and effective chaos bandwidths of the SL as a function of the frequency detuning $\Delta f_{2}$. The results are similar to those of the two VCSELs system. The minimum bandwidth of the chaotic signal from the SL is around zero frequency. Also the effective chaos bandwidth starts to saturate for higher absolute frequency detunings $\Delta \mathrm{f}_{2}$. Figs. 4(c), (d) and (e) are for the $C_{p}$, the $R_{c}$, and the $R_{p p}$, respectively. It is obvious that the TD signature corresponding to the IL varies with the frequency detuning despite that the IL has the same operating parameters over these frequency detunings. This is because the TD signature of the chaotic signal is a statistical result, there is always some fluctuation. It should be stated that the time traces of the IL and SL are recorded simultaneously in the following experimental results. In Fig. 4(c), the peak values $C_{p}$ of the SL around zero frequency detuning and large frequency detuning 
are comparable to those of the IL. The peak to background ratios $\left(R_{c}\right)$ of the SL have the same trend as $C_{p}$, as shown in Fig. 4(d). To know if the TD signature has been totally concealed, the peak to side-peak ratios $\left(R_{p p}\right)$ have been calculated, as shown in Fig. 4(e). It shows that the values of the $R_{p p}$ of the IL during the operation period are all above 1 , so the TD signature of the IL can be identified from the autocorrelation coefficient curve. The TD signatures of the SL are suppressed for most of the frequency detuning, however, $\mathrm{R}_{\mathrm{pp}}$ is still higher than 1 for significant frequency detuning regimes. So using narrow bandwidth optical injection in three-cascaded VCSELs does not shown much advantage in the TD signature concealment compared to the two laser system.

\section{2) Case II - intermediate bandwidth and low TD signature of} the injecting chaos

When the IL operates at point B in Fig. 3(a) and (d), its conventional bandwidth has increased to about $3.4 \mathrm{GHz}$, which is approximately 1.4 times the minimum bandwidth. The power spectrum of the IL is shown in Fig. 2(d2). The TD signature and bandwidth of the SL subject to optical injection from the IL are shown in Fig. 5. For the conventional bandwidth, the trend of the bandwidth variation is similar to that of the IL, as shown in Fig. 5(a). However, for the effective bandwidth, the chaos bandwidth starts to saturate when the frequency detuning is less than $-6.1 \mathrm{GHz}$. The saturated effective bandwidth is about 6 $\mathrm{GHz}$, which is lower than the one for SL subject to narrow bandwidth optical injection. Figs. 5(b), (c) and (d) are for the $C_{p}$, the $R_{c}$, and the $R_{p p}$, respectively. In Fig. 5(b), the peak values $C_{p}$ of the $S L$ at large frequency detunings are comparable to those of the IL. However, the peak to background ratios $\left(R_{c}\right)$ of the SL have decreased in the whole frequency detuning range, as shown in Fig. 5(c). As shown in Fig. 5(d), the TD signature of the IL is relative low; their peak to side-peak ratios are just above 1 , which is the threshold of concealment. With the injection of this lower TD signature chaos into the SL, as expected, the TD signature is suppressed further in the SL. In the whole frequency detuning range, the $\mathrm{R}_{\mathrm{pp}}$ of the $\mathrm{SL}$ are smaller than 1 , which means that it will be difficult for an eavesdropper to identify which peak is the TD signature in the chaotic optical communication, therefore the TD signature has been totally concealed. Fig. 5 demonstrates that bandwidth enhanced chaos with total TD concealment has been obtained at high frequency detuning.
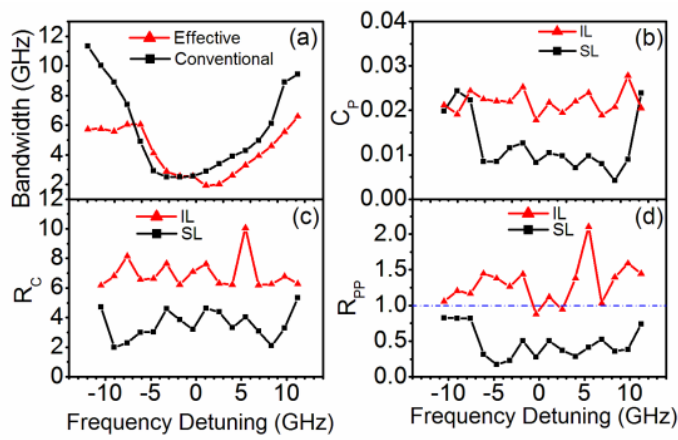

Fig. 5 Three VCSELs system with intermediate bandwidth and lower TD signature of the injecting chaos. (a) conventional and effective chaos bandwidths of the SL, (b-d) the TD signature of the chaotic signal as a function of the frequency detuning $\Delta \mathrm{f}_{2}$ between IL and SL. (b) $\mathrm{C}_{\mathrm{p}}$; (c) $\mathrm{R}_{\mathrm{c}}$; (d) $\mathrm{R}_{\mathrm{pp}}$.

The above results show that the intermediate bandwidth optical injection has better performance in the TD signature concealments compared to the narrow bandwidth chaotic optical injection. As described in [36], the TD concealment may be attributed to the interaction of optical injection and the relaxation oscillation of lasers. For chaotic optical injection, it can be considered as optical injection from many frequency components. The intermediate bandwidth optical injection has more frequency components compared to the narrow bandwidth optical injection, so the TD concealment is better.

\section{3) Case III - broad bandwidth and low TD signature of the injecting chaos}

In the third case, the SL is subject to optical injection from a relative higher bandwidth and lower TD signature chaos. The conventional bandwidth of chaos generated in the IL is about 5.3 GHz, as shown in point $\mathrm{C}$ in Fig. 3(a) and (d). Fig. 6(a) shows the power spectrum of the injecting chaotic signal. The power spectrum is reasonably flat except the lower frequencies. Fig. 6(b) shows both the conventional and effective bandwidth of the chaotic signal from the SL as a function of the frequency detuning $\Delta \mathrm{f}_{2}$. The conventional chaos bandwidth of the SL has the same minimum conventional bandwidth at around zero frequency detuning with a value of about $2.4 \mathrm{GHz}$, however, the minimum effective chaos bandwidth has quite lower value of about $0.9 \mathrm{GHz}$, which means that most of the power is concentrated in a few frequency components. It is noted that the conventional chaotic bandwidth abruptly increases at frequency detuning of $-3.2 \mathrm{GHz}$ in Fig. 6(b). Also the effective bandwidth of the chaos is much lower than those conventional bandwidth of chaos. The reason for these phenomena will be discussed in the next paragraph in conjunction with Fig. 7. Fig. 6(c), (d) and (e) show the $C_{p}, R_{c}$ and $R_{p p}$ as functions of the frequency detuning $\Delta f_{2}$, respectively. From Fig. 6(c), we can see that the SL and IL have the same peak values of autocorrelation coefficient at the TD for most frequency detuning range, except for the frequency detuning around $-4.5 \mathrm{GHz}$ regime. For the frequency detuning around $-4.5 \mathrm{GHz}$, the $\mathrm{C}_{\mathrm{p}}$ values of the $\mathrm{SL}$ are higher than those of the IL. This phenomenon will be explained later. However, the $\mathrm{R}_{\mathrm{c}}$ values of the $\mathrm{SL}$ are comparable to those of the IL, and $\mathrm{R}_{\mathrm{pp}}$ values of the $\mathrm{SL}$ are lower than those of the IL, as shown in Fig. 6(c) and (d). From the TD signature concealment point of view, the SL conceals the TD signature better than the IL. Also $\mathrm{R}_{\mathrm{pp}}$ are lower than 1 for all the frequency detuning except for $\Delta \mathrm{f}_{2}=5.0 \mathrm{GHz}$.

To better understand why the conventional bandwidth increases abruptly at the frequency detuning of $-3.2 \mathrm{GHz}$ and the effective bandwidth of the chaos is much lower than the conventional bandwidth of chaos, the RF power spectra of the SL at different detuning frequencies have been examined and plotted in Fig. 7. We can see that the RF power spectra are very uneven. For the frequency detuning of zero and small negative frequency detuning, the RF power spectra have a single narrow peak. For most of the other frequency detunings, the power 
spectra of the SL have two peaks, which are similar to the experimental result for DFB laser [36]. The peaks at the lower and higher frequencies are considered to be the relaxation oscillation frequency and the beat frequency between the IL and SL, respectively. From the definition of the conventional chaos bandwidth, the chaos bandwidth is slightly higher than the peak frequency for single peak spectra, however, for double peaks spectra, the peak at the lower frequency does not contain $80 \%$ of the total power, the bandwidth will be around the second peak frequency. So, the abrupt increase of the chaotic bandwidth at the frequency detuning of $-3.2 \mathrm{GHz}$ is attributed to the conversion of the single peak of the chaos spectrum to double peaks spectrum. From the definition of the effective chaos bandwidth, the $80 \%$ of the total power is distributed in one peak or two narrow peaks, so the effective bandwidth in case III is much lower than the conventional bandwidth. It is also noted that in the case of single peak spectrum, the peak frequency is higher than the relaxation oscillation frequency. The reason is still unknown. A more detailed study on this aspect will be conducted in the future.

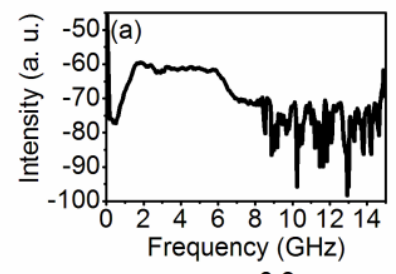

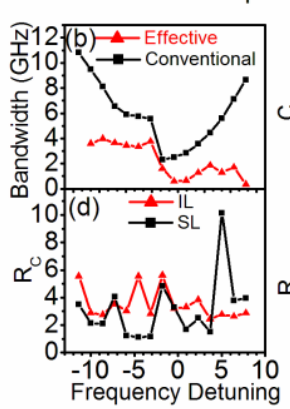

$(\mathrm{GHz})$

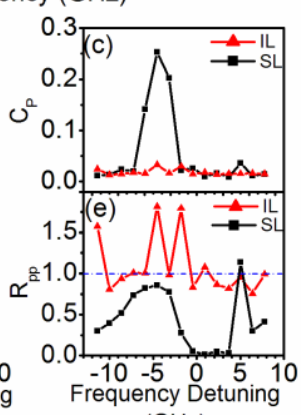

$(\mathrm{GHz})$
Fig.6 Three VCSELs system with broad bandwidth and lower TD signature of the injecting chaos. (a) The RF power spectrum of the IL; (b) conventional and effective chaos bandwidths of the SL and (c-e) the TD signature of the chaotic signal as a function of the frequency detuning $\Delta \mathrm{f}_{2}$ between IL and SL. (c) $\mathrm{C}_{\mathrm{P}}$; (d) $\mathrm{R}_{\mathrm{c}}$; (e) $\mathrm{R}_{\mathrm{PP}}$.

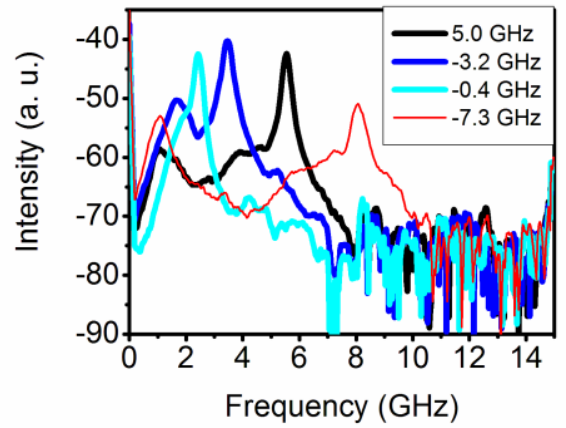

Fig. 7 RF power Spectra of the SL. The thick black, thick dark grey (blue in color version), thick light grey (cyan in color version) and thin grey (red in color version) curves are for the frequency detunings $\Delta \mathrm{f}_{2}$ of $5.0 \mathrm{GHz},-3.2 \mathrm{GHz}$, $-0.4 \mathrm{GHz}$ and $-7.3 \mathrm{GHz}$, respectively.
The reason for the $C_{p}$ values of the SL being higher than those of the IL at frequency detuning around $-4.5 \mathrm{GHz}$ has also been investigated. The power spectrum at the frequency detuning of $-4.5 \mathrm{GHz}$ is similar to that at frequency detuning of $-3.2 \mathrm{GHz}$, as shown in Fig. 7. Most power of the chaotic signal is contained around the frequencies of $1 \mathrm{GHz}$ and $5.5 \mathrm{GHz}$. The autocorrelation coefficient curve and time series of the SL at this frequency detuning are shown in Fig. 8. The autocorrelation coefficient in Fig. 8(a) shows that the TD signature is not significant compared to the background, however the absolute value of $\mathrm{C}_{\mathrm{p}}$ is quite high. All autocorrelation coefficients are large within 120ns delay time, which means that the chaos is not complex. Fig. 8(b) shows the time series of the SL. The inset is the time series in a shorter time interval. The result shows that two dynamics coexist at this frequency detuning. One dynamic has higher amplitude and another one with lower amplitude, which also confirms that the chaos is not very complex. This kind of chaos is not suitable for the applications mentioned in the introduction. The reason for low complexity chaos generation with the high bandwidth chaos injection can be attributed to the lower power density of the injecting beam.

It is also worth pointing out that $R_{p p}$ is only suitable to identify the TD signature with the flat autocorrelation coefficient curve. If the autocorrelation coefficient curve is not flat, as in Fig 8(a), it could be possible to have a clear TD signature peak, but $R_{p p}<1$.
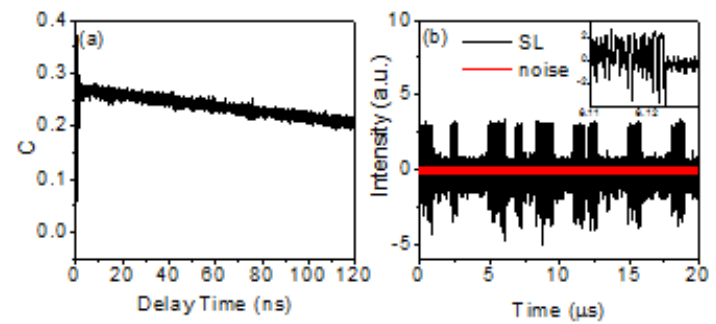

Fig. 8 The autocorrelation coefficient curve and time series of the SL at the frequency detuning of $-4.5 \mathrm{GHz}$. The inset in Fig. 8(b) is the time series in a shorter time interval.

Both similarities and differences can be found by comparing our experimental results with the results in a cascade-coupled DFB lasers system [36] and the numerical simulation results of polarization-resolved chaos in three-cascaded VCSELs [37]. The two-peak structure in the power spectrum of the SL in our experimental result is similar to the experimental result in the DFB system. The experimental results also indicate that the TD signature in the SL has been further suppressed for most of the frequency detuning range in the three cascaded lasers system for all three optical injection cases. Especially for case II (the injecting chaos has medium bandwidth and low TD signature), where the bandwidth enhanced chaos with total TD signature concealment has been achieved over a wider frequency detuning. In [37], the evolutions of TD signature and chaos bandwidth of $\mathrm{X}$-and Y-polarization are very similar, so we expect similar behavior in the total power. Our experimental results confirm that the TD signature can be totally concealed 
over a wide frequency detuning regime, which is similar to the prediction for the polarization-resolved output of the SL [37].

\section{CONCLUSION}

The bandwidth and time-delay signature of chaos generated in three-cascaded VCSELs have been experimentally studied. Three cases of chaotic optical injection have been investigated. In the first case, the injecting chaos has low bandwidth of the chaotic injecting signal and intermediate TD signature. The bandwidth of the chaotic SL as a function of the frequency detuning is similar to that of two lasers system. Also the TD signature cannot be totally concealed for some frequency detuning. For the second case of optical injection, the injecting chaos has an intermediate bandwidth and lower TD signature. The maximum effective bandwidth of the SL is smaller than that for the first case, however, the TD signature of the chaotic signal from the SL can be totally concealed over wide frequency detuning regime, which is similar to the numerical simulation for the polarization-resolved VCSELs output [37]. For the third case of optical injection, the injecting chaos has a high bandwidth and relatively low TD signature. Although the peak to side-peak ratios have decreased to less than 1 for most of the frequency detuning regime, the effective bandwidth have been reduced significantly. The chaos generated by broad bandwidth chaos injection is not very complex for some frequency detuning, which is not suitable for the applications of chaos.

\section{REFERENCES}

[1] C. Masoller, "Anticipation in the synchronization of chaotic semiconductor lasers with optical feedback," Phys. Rev. Lett., vol. 86, no. 13, pp. 2782-2785, 2001.

[2] Y. Takiguchi, K. Ohyagi, and J. Ohtsubo, "Bandwidth-enhanced chaos synchronization in strongly injectionlocked semiconductor lasers with optical feedback," Opt. Lett., vol. 28, no. 5, pp. 319-321, 2003.

[3] A. Argyris, D. Syvridis, L. Larger, V. Annovazzi-Lodi, P. Colet, I. Fischer, J. García-Ojalvo, C. R. Mirasso, L. Pesquera, and K. A. Shore, "Chaos-based communications at high bit rates using commercial fiber-optic links," Nature, vol. 438, no. 7066, pp. 343-346, 2005.

[4] M. Sciamanna, I. Gatare, A. Locquet, and K. Panajotov, "Polarization synchronization in unidirectionally coupled vertical-cavity surface-emitting lasers with orthogonal optical injection," Phys. Rev. E, vol. 75 , no. 5, art. no. $056213,2007$.

[5] Y. Hong, M. W. Lee, J. Paul, P. S. Spencer and K. A. Shore, "GHz bandwidth message transmission using chaotic vertical-cavity surface-emitting lasers", IEEE J. Lightwave Technol., vol. 27, pp.5099-5105, 2009

[6] J.-G. Wu, Z.-M. Wu, G.-Q. Xia, T. Deng, X.-D. Lin, X. Tang and G.-Y. Feng, "Isochronous synchronization between chaotic semiconductor lasers over 40-km fiber links," IEEE Photon. Technol. Lett., vol. 23, no. 24, pp. 1854-1856, Dec. 2011

[7] S. Y. Xiang, W. Pan, B. Luo, L. S. Yan, X. H. Zou, N. Jiang, N. Q. Li, and H. N. Zhu, "Message encoding/decoding using unpredictability-enhanced chaotic VCSELs," IEEE Photon. Technol. Lett., vol. 24, no. 15, pp. 1268-1269, Aug. 2012

[8] A. Uchida, K. Amano, M. Inoue, K. Hirano, S. Naito, H. Someya, I. Oowada, T. Kurashige, M. Shiki, S. Yoshimori, K. Yoshimura, and P. Davis, "Fast physical random bit generation with chaotic semiconductor lasers," Nat. Photon., vol. 2, no. 12, pp.728-732, 2008.

[9] I. Kanter, Y. Aviad, I. Reidler, E. Cohen, and M. Rosenbluh, “An optical ultrafast random bit generator," Nat. Photon., vol. 4, no. 1, pp. 58-61, 2010.

[10] K. Hirano, T. Yamazaki, S. Morikatsu, H. Okumura, H. Aida, A. Uchida, S. Yoshimori, K. Yoshimura, T. Harayama, and P. Davis. (2010, Mar.).
Fast random bit generation with bandwidth-enhanced chaos in semiconductor lasers. Opt. Express [Online]. 18(6), pp. 5512-5524. Available:

http://www.opticsinfobase.org/oe/abstract.cfm?URI=oe-18-6-5512

[11] A. Wang, N. Wang, Y. Yang, B. Wang, M. Zhang, and Y. Wang, "Precise Fault Location in WDM-PON by Utilizing Wavelength Tunable Chaotic Laser", IEEE J. Lightwave Technol., vol. 30, no. 21, pp. 3420-3426, Nov. 2012.

[12] L. Xia, D. Huang, J. Xu, and D. Liu, "Simultaneous and precise fault locating in WDM-PON by the generation of optical wideband chaos," Opt. Lett., vol. 38, pp. 3762-3764, 2013.

[13] Z. N. Wang, M. Q. Fan, L. Zhang, H. Wu, D. V. Churkin, Y. Li, X. Y. Qian, and Y. J. Rao. (2015, June). Long-range and high-precision correlation optical time-domain reflectometry utilizing an all-fiber chaotic source. Opt. Express [Online]. 23(12), pp. 15514-15520. Available: https://www.osapublishing.org/oe/abstract.cfm?uri=oe-23-12-15514

[14] F. Y. Lin and J. M. Liu, "Chaotic lidar," IEEE J. Sel. Topics Quantum Electron., vol. 10, no. 5, pp. 991-997, Sep. 2004.

[15] R. Roy and K. S. Thornburg Jr., "Experimental synchronization of chaotic lasers," Phys. Rev. Lett., vol. 72, pp. 2009-2012, 1994.

[16] T. Sugawara,M. Tachikawa, T. Tsukamoto, and T. Shimisu, "Observation of synchronization in laser chaos," Phys. Rev. Lett., vol. 72, pp. 35023506, 1994.

[17] G. D. VanWiggeren and R. Roy, "Communication with chaotic lasers," Science, vol. 279, pp. 1198-1200, 1998.

[18] M. Sciamanna and K. A. Shore, "Physics and applications of laser diode chaos," Nature Photonics, vol. 9, no.3, pp.151-162, March 2015.

[19] M. Virte, K. Panajotov, H. Thienpont and M. Sciamanna, "Deterministic polarization chaos from a laser diode", Nature Photonics, vol. 7, pp. 6065, 2013.

[20] M. Virte, E. Mercier, H. Thienpont, K. Panajotov, M. Sciamanna, [2014, July]. Physical random bit generation from chaotic solitary laser diode. Opt. Express [Online], 22(14), pp. 17271-17280. Available: https://www.osapublishing.org/oe/abstract.cfm?uri=oe-22-14-17271

[21] G. P. Agrawal, "Effect of Gain Nonlinearities on Period Doubling and Chaos in Directly Modulated Semiconductor Lasers," Appl. Phys. Lett., vol. 49, no.16, pp. 1013-1015, 1986.

[22] A. Uchida, T. Heil, Y. Liu, P. Davis, and T. Aida, "High-frequency broadband signal generation using a semiconductor laser with a chaotic optical injection," IEEE J. Quantum Electron., vol. 39, no. 11, pp. $1462-$ 1467, Nov. 2003.

[23] Y. Hong, P. S. Spencer, and K. A. Shore, "Enhancement of chaotic signal bandwidth in vertical-cavity surface-emitting lasers with optical injection," J. Opt. Soc. Amer. B, vol. 29, no. 3, pp. 415-419, Mar. 2012.

[24] S. Y. Xiang, W. Pan, B. Luo, L. S. Yan, X. H. Zou, N. Li, and H. N. Zhu, "Wideband unpredictability-enhanced chaotic semiconductor lasers with dual-chaotic optical injections," IEEE J. Quantum Electron., vol. 48, no. 8, pp. 1069-1076, Aug. 2012.

[25] A. B. Wang, Y. C. Wang, Y. B. Yang, M. J. Zhang, H. Xu, and B. J. Wang, "Generation of flat-spectrum wideband chaos by fiber ring resonator," Appl. Phys. Lett., vol. 102, no. 3, pp. 031112-1-031112-5, 2013.

[26] Y. Hong, "Flat broadband chaos in mutually coupled vertical-cavity surface-emitting lasers", IEEE J. Select. Top. Quantum. Electron. vol. 21, no. 6, art. no. 1801007, Nov./Dec. 2015.

[27] D. Rontani, A. Locquet, M. Sciamanna, and D. S. Citrin, "Loss of time-delay signature in the chaotic output of a semiconductor laser with optical feedback," Opt. Lett., vol. 32, no. 20, pp. 2960-2962, Oct. 2007

[28] R. M. Nguimdo, P. Colet, L. Larger, and L. Pesquera, "Digital key for chaos communication performing time delay concealment," Phys. Rev. Lett., vol. 107, no. 3, pp. 034103-1-034103-4, Jul. 2011.

[29] J.-G. Wu, Z.-M. Wu, G.-Q. Xia, and G.-Y. Feng. [2012, Jan]. Evolution of time delay signature of chaos generated in a mutually delay-coupled semiconductor lasers system. Opt. Express [Online]. 20(2), pp. 17411753. https://www.osapublishing.org/oe/abstract.cfm?uri=oe-20-2-1741

[30] S.-S. Li, Q. Liu, and S.-C. Chan. [2012, Oct.]. Distributed feedbacks for time-delay signature suppression of chaos generated from a semiconductor laser. IEEE Photon. Journal [Online], 4( 5), pp. 19301935. Available: http://ieeexplore.ieee.org/xpls/abs_all.jsp?arnumber=6320711\&tag=1

[31] Y. Hong. [2013, Jul.]. Experimental study of time-delay signature of chaos in mutually coupled vertical-cavity surface-emitting lasers subject to polarization rotated optical injection. Opt. Express [Online], 21(15), 
pp.17894-17903.

Available: http://www.opticsinfobase.org/oe/abstract.cfm?uri=oe-21-15-17894

[32] H. Lin, Y. Hong and K. A. Shore, "Experimental study of time-delay signatures in vertical-cavity surface-emitting lasers subject to double-cavity polarization-rotated optical feedback", IEEE J. Lightwave Technol., vol. 32, no. 9, pp. 1829-1836, May 2014.

[33] A. B. Wang, Y. B. Yang, B. J. Wang, B. B. Zhang, L. Li, and Y. C. Wang [2013, April]. Generation of wideband chaos with suppressed time-delay signature by delayed self-interference. Opt. Express [Online], 21(7), pp. 8701-8710. https://www.osapublishing.org/oe/abstract.cfm?uri=oe-21-7-8701

[34] Y. Hong, P. S. Spencer, and K. A. Shore, "Wideband chaos with time delay concealment in vertical-cavity surface-emitting lasers with optica feedback and injection," IEEE J. Quantum Electron., vol. 50, no. 4, pp. 236-242, 2014.

[35] C.-H. Cheng, Y.-C. Chen and F.-Y. Lin. [2015, Feb.]. Chaos time delay signature suppression and bandwidth enhancement by electrical heterodyning. Opt. Express [Online], 23(3), pp.2308-2319. Available: https://www.osapublishing.org/oe/abstract.cfm?uri=oe-23-3-2308

[36] N. Li, W. Pan, S. Xiang, L. Yan, B. Luo, and X. Zou, "Loss of time delay signature in broadband cascade-coupled semiconductor lasers," IEEE Photon. Technol. Lett., vol. 24, no. 23, pp.2187-2192, Dec. 2012

[37] H. Liu, N. Li, and Q. Zhao, "Photonic generation of polarization-resolved wideband chaos with time-delay concealment in three-cascaded vertical-cavity surface-emitting lasers" Appl. Opt., vol. 54, no. 14, pp. 4380-4386, 2015.

[38] S. Xiang, W. Pan, B. Luo, L. Yan, X. Zou, N. Jiang, L. Yang, and H. Zhu, "Conceal time-delay signature of chaotic vertical-cavity surface-emitting lasers by variable-polarization optical feedback," Opt. Commun., vol. 284, pp. 5758-5765, 2011.

[39] R. M. Nguimdo, G. Verschaffelt, J. Danckaert, and G. Van der Sande, "Loss of time-delay signature in chaotic semiconductor ring lasers," Opt. Lett., vol. 37, no. 13, pp. 2541-2543, July 2012.

[40] R. M. Nguimdo, M. C. Soriano, and P. Colet, "Role of the phase in the identification of delay time in semiconductor lasers with optical feedback," Opt. Lett., vol. 36, no. 22, pp. 4332-4334, Nov 2011.

[41] C. Bandt and B. Pompe, "Permutation entropy: a natural complexity measure for time series," Phys. Rev. Lett. vol. 88, art. no. 174102, 2002

[42] F.-Y. Lin, Y.-K. Chao, and T.-C. Wu, "Effective bandwidths of broadband chaotic signals," IEEE J. Quantum Electron., vol. 48, no.8, pp. 1010-1014, Aug. 2012.

Yanhua Hong received the B.Sc. degree in physics from Fujian Normal University, Fuzhou, China, the M.S. degree in physics from Beijing Normal University, Beijing, China, and the Ph.D. degree in optics from the Institute of Physics, Chinese Academy of Sciences, Beijing, in 1987, 1990, and 1993, respectively.

She was a Lecturer with Beihang University, Beijing, from 1993 to 1997. Since 1997, she has been with Bangor University, Bangor, U.K., where she became a Permanent Research Staff in 2007, Lecturer in 2013 and Senior Lecturer in 2016. She is the author or co-author of more than 160 journal and conference papers. Her current research interests include nonlinear dynamics in edge-emitting semiconductor lasers, vertical-cavity surface-emitting lasers, semiconductor optical amplifiers, and optical communication systems based on optical orthogonal frequency division multiplexing.

Dr. Hong is a member of IEEE and a senior member of the Optical Society of America

Ana Quirce received the Licenciada en Física (M.Sc.) degree and the Ph.D. degree in sciences, technologies, and computing from the University of Cantabria, Santander, Spain, in 2008 and 2012, respectively, where she is studying the dynamics of the polarization and transverse modes of vertical cavity surface-emitting lasers (VCSELs) subject to optical injection.

She is currently a Post-Doctoral Researcher with the Vrije Universiteit Brussel, Brussels, Belgium. Her current research interests are in the areas of dynamics of VCSELs, optical injection effects in semiconductor lasers, and optical feedback.

Bingjie Wang received the Ph.D. degree in electronic circuit and system from Taiyuan University of Technology, Taiyuan, China, in 2012. She is currently an Assistant Professor with the College of Physics and Optoelectronics, Taiyuan University of Technology. Her current research interests include laser dynamics and wire health monitoring using chaotic signal.
Songkun Ji received the B.A. degree in physics in State University of New York at Geneseo in 2013 and the M.Sc. degree in Electronic Engineering in Bangor University in 2015. He is currently working toward the Ph.D. degree in Electronic Engineering.

His current research interests are in the areas of nonlinear dynamics of vertical-cavity surface-emitting lasers subject to optical injection and optical feedback and photonic microwave generation.

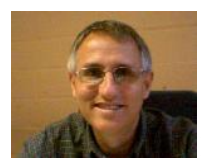

Krassimir Panajotov received the B.S., Ph.D., and D.Sc. degrees in physics from Sofia University, Sofia, Bulgaria, in 1982, 1988, and 2002 , respectively. Since 1982, he has been appointed at the Institute of Solid State Physics (ISSP), Bulgarian Academy of Sciences. During 1996-1998, he was a Guest Researcher at the Vrije Universiteit Brussels (VUB), Brussels, Belgium, which he joined again in 2000. His research activities are in nonlinear optics, semiconductor lasers, photonic crystal devices, and nonlinear semiconductor laser dynamics. In these fields, he holds more than 170 SCI-stated journal papers, more than 180 publications in international conference proceedings and 7 book chapters. Prof. Panajotov has led a number of projects on national level in Bulgaria and Belgium and international bilateral projects and served as a national coordinator of several European COST actions. He co-chaired the conferences on Semiconductor Laser and Laser Dynamics at the Photonics Europe'2008 - , '2016 and five International Workshops. He has served as a guest editor of 4 Special Issues of Optical and Quantum Electronics and Advances in Optics Technologies, as well as of 5 SPIE proceedings. He is currently associated editor of Journal of Opto-Electronics Review. Prof. Panajotov is a member of IEEE and a Senior member of OSA.

Paul S. Spencer was born in England and received both his B.Sc. degree in Applied Physics and his Ph.D. in Electronic Engineering from the University of Bath, U.K. Following post-doctoral experience at several universities, he was appointed to a lectureship in the School of Electronic Engineering and Computer Science at Bangor University. He was subsequently promoted to senior lecturer and in 2006 he was awarded a Personal Chair within the School of Electronic Engineering. He was Head of School for several years and is currently the Dean of the College of Physical and Applied Sciences at Bangor University.

In 2000 he won the Science, Engineering and Technology Award for best engineering poster at the annual meeting held in the House of Commons, London. He is a Fellow of the Institute of Physics and has been the Honorary Treasurer of the Institute of Physics (IoP) Semiconductor Physics Group. He is regularly a session chair at both national and international workshops and conferences. He has also provided invited contributions to several books: 'Recent Advances in Laser Dynamics: Control and Synchronistation,' Editor A.N.Pisarchik, Research Signpost, 2008, 'Handbook of Chaos Control, Second Edition, Editors: Eckehard Scholl, and Heinz Georg Schuster, Wiley-VCH, (2007) and 'Unlocking Dynamical Diversity: Optical Feedback Effects on Semiconductor Lasers', Editors: D.M. Kane and K.A. Shore, Wiley, (2005).

He has published over 100 archival journal publications and nearly 200 conference papers. He has also been either the principal investigator or co-investigator on numerous EU and national research grants. His main research interests are in the dynamics of semiconductor lasers and amplifiers and optical pulse propagation, through optoelectronic devices. Recent work has focussed on the theoretical modelling of complex nonlinear dynamical systems and in particular on the control and use of chaotic waveforms. 